\title{
Two-time-scale MPC for Economically Optimal Real-time Operation of Balance Responsible Parties *
}

\author{
Panagiotis Patrinos* Daniele Bernardini* \\ Alessandro Maffei ${ }^{* *}$ Andrej Jokic ${ }^{* * *}$ Alberto Bemporad* \\ * IMT Institute for Advanced Studies Lucca, Piazza San Ponziano 6, \\ 55100 Lucca, Italy (e-mail: panagiotis.patrinos@imtlucca.it, \\ daniele.bernardini@imtlucca.it, alberto.bemporad@imtlucca.it) \\ ** University of Trento, Via Mesiano 77, 38100, Italy (e-mail: \\ a.maffei@studenti.unitn.it) \\ *** Eindhoven University of Technology, PO Box 513, 5600 MB \\ Eindhoven, The Netherlands (e-mail: a.jokic@tue.nl)
}

\begin{abstract}
European electrical networks are evolving towards a distributed system where the number of power plants is growing and also the green plants based on renewable energy sources (RES) like wind and solar are increasing. Integration of RES leads to energy imbalance, due to the difficulty to predict their production. This paper proposes a two-time-scale Hierarchical Model Predictive Control (HMPC) strategy for real-time optimal control of Balance Responsible Parties (BRPs) in power systems with high penetration of renewable energy sources (RES). The proposed control strategy is able to handle ramp-rate constraints efficiently and results in reduced generation and imbalance costs due to real-time economic optimization of power setpoints.
\end{abstract}

Keywords: Smart grid; Model predictive control; Hierarchical control.

\section{INTRODUCTION}

Liberalization and deregulation of electricity markets have led to a competitive environment consisting of market participants (usually termed as Balance Responsible Parties) that are legally entitled to trade electricity on the various markets in order to satisfy their loads and make profits. On the other hand, adoption levels of renewable resources are continuously increasing due to the need for a decrease of production costs and greenhouse emissions from electricity generation by conventional fossil-fueled power plants (e.g., coal, gas, etc.). Efficient integration of intermittent generation into the existing power grid is a major bottleneck due to high variability and low predictability of renewable resources, especially wind [Ummels et al., 2007].

Although market structures vary with respect to each country, they share some common characteristics. Specifically, in the majority of electricity markets, participant BRPs place their bids on the Day-Ahead (DA) market and the Ancillary Services (AS) market regarding energy delivery and capacity availability for each Program Time Unit (PTU) of the following day. At the end of the dayahead auction, the Transmission System Operator (TSO) selects the accepted and rejected bids according to some clearing mechanism [Shahidehpour et al., 2002] and publishes the future prices and volumes, for each PTU of the following day. Subsequently, each BRP determines its

\footnotetext{
* This work was partially by the European project E-PRICE: Pricebased Control of Electrical Power Systems, FP7-IST contract no. 249096
}

Energy Program (E-Program) and sends it to the TSO for approval. The latter describes the amount of energy supplied or consumed by the BRP at every Program Time Unit (PTU) of the following day. After receiving approval by the TSO, each BRP executes a unit commitment module to determine the on/off status of its generators for each PTU of the following day.

Due to uncertainties in power demand and generation, the existence of an imbalance market (or real-time market) operated by the TSO is mandatory in order to counteract real-time global energy imbalances [Jokic, 2007]. Unlike day-ahead prices, imbalance prices are characterized by large volatility, sudden spikes that are hard to predict, and counterintuitive phenomena like negative values. Negative prices result from positive imbalance, where supply is larger than demand, especially during the night [Sewalt and de Jong, 2003]. In real-time, a BRP must fulfill its EProgram in order to avoid internal imbalance and imbalance costs, and it has to cope with uncertainties induced by intermittent generation from renewable sources, timevarying loads and imbalance prices, as well as perturbations of its E-Program due to AS bids activated by the TSO. If the TSO calls for a specific AS bid, the BRP responsible for this bid is asked to deliver the requested energy, by adjusting its E-Program accordingly. The realtime control problem is further complicated by the coupling of energy between consecutive PTUs due to the ramp-rate constraints. 
In this paper we propose a two-time-scale Hierarchical Model Predictive Control (HMPC) algorithm for efficient real-time operation of a BRP. The upper-level MPC computes power and energy setpoints based on predictions of the uncertain exogenous inputs (AS bids activated by TSO, imbalance prices, intermittent generation and load) by minimizing BRP costs. The lower-level MPC tries to track the set-points received by upper-level MPC by taking into account the detailed dynamics of the generators and operating on a refined time-scale. The goal of the controller is to determine power set-points for the controllable generators so as to minimize generation costs (utilize intermittent resources as much as possible) and economically track the deviated E-Program, meaning that it may be profitable sometimes to deviate either upwards or downwards depending on the imbalance price. The main advantages of the proposed scheme are the improved performance due to the real-time calculation of economically optimal setpoints, the integral action with respect to the E-Program due to energy tracking, and the effective handling of ramprate constraints which allows smooth transitions between PTUs. The effefctiveness of the proposed approach is exemplified in a case study which emulates the Dutch power system.

\section{NOTATION}

Let $\mathbb{R}, \mathbb{R}^{n}, \mathbb{R}^{m \times n}$ denote the field of real numbers, the set of non-negative integers, the set of column real vectors of length $n$ and the set of $m$ by $n$ real matrices, respectively. The transpose of a matrix $A \in \mathbb{R}^{m \times n}$ is denoted by $A^{\prime}$. For any nonnegative integers $k_{1} \leq k_{2}$, the finite set $\left\{k_{1}, \ldots, k_{2}\right\}$ is denoted by $\mathbb{N}_{\left[k_{1}, k_{2}\right]}$. For $x \in \mathbb{R}, y \neq 0,\lfloor x\rfloor$ is the greatest integer smaller than or equal to $x$ and $\bmod \frac{x}{y} \triangleq x-y\left\lfloor\frac{x}{y}\right\rfloor$. The direct sum of matrices $A_{i} \in$ $\mathbb{R}^{n_{i} \times m_{i}}, i \in \mathbb{N}_{\left[k_{1}, k_{2}\right]}$ is denoted by $\bigoplus_{i=k_{1}}^{k_{2}} A_{i}$. If $x \in \mathbb{R}^{n}$ and $Q \in \mathbb{R}^{n \times n}$ is positive definite, then $\|x\|_{Q}^{2} \triangleq x^{\prime} Q x$.

\section{BASIC SETUP}

We consider a BRP consisting of $n_{\mathrm{p}}$ controllable generators, $n_{\mathrm{r}}$ uncontrollable generators and uncontrollable load. The basic information needed for the setup is the E-Program and generators schedule resulting from the unit commitment problem solved the day before, for every PTU. The goal of the control strategy to be developed is to optimize the economic performance of the BRP, by minimizing production costs and imbalance charges while hedging against uncertainties due to imbalance prices, intermittent generation, loads and E-Program perturbations.

For that purpose we propose a HMPC algorithm based on a temporal decomposition of the problem in two time scales (energy and power). Specifically, the upper level MPC scheme operates on the energy time-scale, whose sampling period is less than or equal to the length of a PTU and, by optimizing the economic performance of the BRP, it calculates setpoints of average power injections for the controllable generators, as well as energy setpoints based on predictions of the perturbed E-Program, intermittent generation, loads and imbalance prices. The lower level MPC scheme operates on the power time-scale, whose sampling period is less than or equal to that of the energy time scale (usually 4 secs), trying to track the setpoints provided by the upper level MPC while taking into account the detailed dynamics and constraints of the controllable generators.

\subsection{Power and Energy Time Scales}

The length of a PTU is denoted by $T_{\mathrm{PTU}}[\mathrm{s}]$, while $T_{\mathrm{E}}[\mathrm{s}]$ is the sampling time of the energy time-scale and $T_{\mathrm{P}}[\mathrm{s}]$ is the sampling time of the power time-scale. We assume that $T_{\mathrm{PTU}} \geqslant T_{\mathrm{E}} \geqslant T_{\mathrm{P}}$ and that

$$
r_{\mathrm{ET}} \triangleq \frac{T_{\mathrm{PTU}}}{T_{\mathrm{E}}}, r_{\mathrm{PE}} \triangleq \frac{T_{\mathrm{E}}}{T_{\mathrm{P}}}, r_{\mathrm{PT}} \triangleq \frac{T_{\mathrm{PTU}}}{T_{\mathrm{P}}}
$$

are positive integers. We use symbols $n, k$ and $t$ to index time in the PTU, energy and power time scale respectively. For $k \in \mathbb{Z}_{+}$, let $\kappa_{\mathrm{ET}}(k) \triangleq\left\lfloor\frac{k}{r_{\mathrm{ET}}}\right\rfloor$. Then $\kappa_{\mathrm{ET}}(k)=n$, if and only if $k T_{\mathrm{E}} \in\left[n T_{\mathrm{PTU}},(n+1) T_{\mathrm{PTU}}\right)$, i.e., the time instant $k$ on the energy time scale belongs to the $n$-th PTU. Similarly, for $t \in \mathbb{Z}_{+}$, let $\kappa_{\mathrm{PE}}(t) \triangleq\left\lfloor\frac{t}{r_{\mathrm{PE}}}\right\rfloor, \kappa_{\mathrm{PT}}(t) \triangleq\left\lfloor\frac{t}{r_{\mathrm{PT}}}\right\rfloor$. Consider PTU $n \in \mathbb{Z}_{+}$. Then, the intervals on the energy time-scale that are active along $n$, i.e., the indices $k$ such that $\kappa_{\mathrm{ET}}(k)=n$, are $k \in \mathbb{N}_{\left[k_{\min }(n), k_{\max }(n)\right]}$, where $k_{\min }(n) \triangleq r_{\mathrm{ET}} n, k_{\max }(n) \triangleq r_{\mathrm{ET}}(n+1)-1$. Similarly, for $k \in \mathbb{Z}_{+}$in the energy time-scale, the set of intervals on the power time-scale that are active along $k$, are $t \in$ $\mathbb{N}_{\left[t_{\min }(k), t_{\max }(k)\right]}$, where $t_{\min }(k) \triangleq r_{\mathrm{PE}} k, t_{\max }(k) \triangleq r_{\mathrm{PE}}(k+$ $1)-1$. The trajectories of controllable power injections, uncontrollable power injections and load [MW], on the power time scale are denoted by $p^{i}(t), i=1, \ldots, n_{\mathrm{p}}$, $r^{i}(t), i=1, \ldots, n_{\mathrm{r}}, d(t)$, respectively. Furthermore, let $p(t)=\left(p^{1}(t) \cdots p^{n_{\mathrm{p}}}(t)\right), r(t)=\left(r^{1}(t) \cdots r^{n_{\mathrm{r}}}(t)\right)$. The average values of the trajectory of $p^{i}(t)$ within interval $k$ is

$$
p^{i, \mathrm{av}}(k)=\frac{1}{r_{\mathrm{PE}}} \sum_{t=t_{\min }(k)}^{t_{\max }(k)} p^{i}(t), \quad i \in \mathbb{N}_{\left[1, n_{\mathrm{p}}\right]} .
$$

Similar notation is used for $r^{i}(t), d(t)$. Let $\bar{p}^{i}(k)$ denote the average controllable power injection as a variable at some future energy time interval $k$. Similarly, let $\bar{r}^{i}(k), \bar{d}(k)$ denote the predicted values of $r^{i, \text { av }}(k), d^{\text {av }}(k)$ respectively, at some future energy time interval $k$. Finally, let $\bar{p}(k)=$ $\left(\bar{p}^{1}(k), \ldots, \bar{p}^{n_{\mathrm{p}}}(k)\right), \bar{r}(k)=\left(\bar{r}^{1}(k) \cdots \bar{r}^{n_{\mathrm{r}}}(k)\right)$.

\subsection{Energy balances}

For $k \in \mathbb{Z}_{+}$and $t \in \mathbb{N}_{\left[t_{\min }(k), t_{\max }(k)+1\right]}$, let $e_{k}^{\mathrm{p}}(t), e_{k}^{\mathrm{rd}}(t)$, $e_{k}(t)[\mathrm{MWh}]$ denote the accumulated actual energy produced by controllable generators, by uncontrollable generators and load, and accumulated actual net energy, respectively, along energy time interval $k$, up to time instant $t T_{\mathrm{P}}$ (during the time interval $\left[t_{\min }(k) T_{\mathrm{P}}, t T_{\mathrm{P}}\right)$ ), based on the actual power injections of the BRP on the power time scale. For $k \in \mathbb{Z}_{+}, e_{k}^{\mathrm{p}} \triangleq e_{k}^{\mathrm{p}}\left(t_{\max }(k)+1\right)$, $e_{k}^{\mathrm{rd}} \triangleq e_{k}^{\mathrm{rd}}\left(t_{\max }(k)+1\right)$ and $\left.e_{k} \triangleq e_{k}\left(t_{\max }(k)+1\right)\right)$ are the accumulated energy produced by controllable generators, by uncontrollable generators and load, and total net energy within the energy time interval $k$, respectively.

Accordingly, for $n \in \mathbb{Z}_{+}$and $k \in \mathbb{N}_{\left[k_{\min }(n), k_{\max }(n)+1\right]}$, let $e^{\mathrm{p}}(n ; k), e^{\mathrm{rd}}(n ; k), e(n ; k)[\mathrm{MWh}]$ denote the accumulated actual energy produced by controllable generators, 
by uncontrollable generators and load, and accumulated actual net energy, respectively, along PTU $n$, up to time instant $k T_{\mathrm{E}}$ (during the time interval $\left[k_{\min }(n) T_{\mathrm{E}}, k T_{\mathrm{E}}\right)$ ), based on the real power injections of the BRP. We denote with $\bar{e}^{\mathrm{p}}(n ; k), \bar{e}^{\mathrm{rd}}(n ; k), \bar{e}(n ; k)$ the emphpredicted values of $e^{\mathrm{p}}(n ; k), e^{\mathrm{rd}}(n ; k), e(n ; k)$, respectively. Finally, for $n \in$ $\mathbb{Z}_{+}, e^{\mathrm{p}}(n) \triangleq e^{\mathrm{p}}\left(n ; k_{\max }(n)+1\right), e^{\mathrm{rd}}(n) \triangleq e^{\mathrm{rd}}\left(n ; k_{\max }(n)+\right.$ $1)$ and $\left.e(n) \triangleq e\left(n ; k_{\max }(n)+1\right)\right)$ denote the accumulated energy produced by controllable generators, by uncontrollable generators and load and total energy respectively, along PTU $n$, while $\bar{e}^{\mathrm{p}}(n), \bar{e}^{\mathrm{rd}}(n), \bar{e}(n)$ denote the predictions of the corresponding quantities.

\section{MPC ON THE ENERGY TIME SCALE}

In the proposed real-time hierarchical MPC scheme, given the E-program and the generator schedule, the upperlevel MPC performs economic optimization deciding the power and energy setpoints for the lower-level MPC, based on predictions for the uncertain load, uncontrollable generation and distorted E-Program, so as to minimize production and imbalance costs.

\subsection{Generation costs}

It is assumed that individual generation costs of controllable generators are given by convex quadratic functions, i.e., $\ell_{i}^{\mathrm{P}}\left(\bar{p}^{i}\right)=a_{i}\left(\bar{p}^{i}\right)^{2}+b_{i} \bar{p}^{i}+c_{i}, a_{i} \geqslant 0, i \in \mathbb{N}_{\left[1, n_{\mathrm{p}}\right]}$. The total generation cost of the BRP consists of the sum of production costs related to the controllable generators, i.e., $\ell^{\mathrm{p}}: \mathbb{R}^{n_{\mathrm{p}}} \rightarrow \mathbb{R}$ is given by $\ell^{\mathrm{p}}(\bar{p}) \triangleq \sum_{i=1}^{n_{\mathrm{p}}} \ell_{i}^{\mathrm{p}}\left(\bar{p}^{i}\right)$.

\subsection{Imbalance costs}

For $n \in \mathbb{Z}_{+}, e^{\operatorname{prog}}(n)$ is the energy [MWh] that the BRP is commited to supply to/absorb from the TSO at PTU $n$, according to its E-Program, determined the day-ahead. On the power time scale (usually 4 secs) the TSO sends to each BRP actively participating in the SC arrangements a delta power signal $\delta p(t)[\mathrm{MW}](<0,=0$ or $>0)$ which the $\mathrm{BRP}$ has to realize by changing the power setpoint of the selected units for SC. Then, the BRP's E-Program is offset with the requested energy resulting in the final E-Program $e^{\text {final }}(n)$ :

$$
e^{\text {final }}(n) \triangleq e^{\operatorname{prog}}(n)+\sum_{\left\{t \in \mathbb{Z}_{+} \mid \kappa_{\mathrm{PT}}(t)=n\right\}} \delta p(t) .
$$

For future use, let

$$
e^{\mathrm{sc}}(k)=\frac{T_{P}}{3600} \sum_{t=t_{\min }(k)}^{t_{\max }(k)} \delta p(t) \quad[\mathrm{MWh}] .
$$

The energy imbalance $\Delta e(n)$ of the $\mathrm{BRP}$ at PTU $n$ is the difference between the actual energy produced by the BRP at PTU $n$ and $e^{\text {final }}(n)$ :

$$
\Delta e(n) \triangleq e(n)-e^{\text {final }}(n) .
$$

If the BRP has a surplus of energy $(\Delta e(n)>0)$, then the TSO buys this energy at imbalance price surplus $\lambda_{\mathrm{IM}}^{+}(n)$ $\left(\lambda_{\mathrm{IM}}^{+}(n)\right.$ can be negative, in which case the BRP is the one who pays the TSO). On the contrary, if the BRP has a shortfall of energy $(\Delta e(n)<0)$, then it buys it from the TSO at imbalance price shortfall $\lambda_{\mathrm{IM}}^{-}(n)\left(\lambda_{\mathrm{IM}}^{-}(n)\right.$ can be negative, in which the TSO is the one who pays the BRP). Therefore, the imbalance cost for the BRP is:

$$
\ell_{\mathrm{IM}}(\Delta e)=-\lambda_{\mathrm{IM}}^{+}[\Delta e]_{+}+\lambda_{\mathrm{IM}}^{-}[-\Delta e]_{+},
$$

where $[x]_{+} \triangleq \max \{x, 0\}$. Since $[x]_{+}=\frac{1}{2}(|x|+x)$, it follows that

$$
\ell_{\mathrm{IM}}(\Delta e)=\frac{1}{2}\left(\lambda_{\mathrm{IM}}^{-}-\lambda_{\mathrm{IM}}^{+}\right)|\Delta e|-\frac{1}{2}\left(\lambda_{\mathrm{IM}}^{-}+\lambda_{\mathrm{IM}}^{+}\right) \Delta e
$$

Here, we make the assumption that $\lambda_{\text {IM }}^{-}-\lambda_{\text {IM }}^{+} \geqslant 0$. The assumption is substantiated by historical data obtained by the Dutch TSO (TenneT), where for imbalance prices of one year, only $0.008 \%$ of the PTUs have $\lambda_{\text {IM }}^{-}-\lambda_{\text {IM }}^{+}<0$. Under this assumption, $\ell_{\mathrm{IM}}$ is a convex piecewise-affine (PWA) function as the nonnegative weighted sum of two convex PWA functions.

\subsection{Upper-level $M P C$}

The goal of the upper-level MPC is to compute power and energy setpoints for the lower-level MPC that minimize production and imbalance costs, while accounting for disturbances due to uncertain intermittent generation, loads and activated AS bids.

Let $N_{\mathrm{E}}$ denote the prediction horizon of the MPC controller operating on the energy time-scale. Then, the PTUs that are active during the prediction horizon, starting from

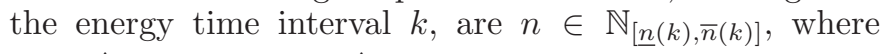
$\underline{n}(k) \triangleq \kappa_{\mathrm{ET}}(k), \bar{n}(k) \triangleq \kappa_{\mathrm{ET}}\left(k+N_{\mathrm{E}}-1\right)$. According to the MPC philosophy, at every $k \in \mathbb{Z}_{+}$on the energy time scale, i.e., at time $k T_{\mathrm{E}}$, we formulate and solve the finitehorizon optimal control problem (6), where the goal is find a sequence of power and energy setpoints for the generators so as to minimize the expected cost along the prediction horizon while satisfying the constraints.

According to (6a), the goal is to find power setpoints that minimize the expected generation and imbalance costs along the energy prediction horizon. (6b) provides the initial conditions for the MPC problem, while (6c) imposes bounds on the power output of each generator. If a generator is off at PTU $\kappa_{\mathrm{ET}}(j)$, then $(6 \mathrm{c})$ enforces $\bar{p}^{i}(j)=0$, since $\mathbf{I}_{i, \kappa_{\mathrm{ET}}(j)}=0$. The power profile of each controllable generator must adhere to ramp-rate constraints dictated by maximum downward and upward ramping limits, $\Delta p_{\min }$, $\Delta p_{\max }[\% / \mathrm{min}]$. This is imposed by equations (6d), (6e). Notice that here we distinguish between two cases. If generator $i$ was on at PTU $\kappa_{\mathrm{ET}}(j-1)\left(\mathbf{I}_{i, \kappa_{\mathrm{ET}}(j-1)}=1\right)$ then the relative ramp-rate is computed in terms of $\bar{p}^{i}(j-1)$. However, if generator $i$ was off during $\operatorname{PTU} \kappa_{\mathrm{ET}}(j-1)$ $\left(\mathbf{I}_{i, \kappa_{\mathrm{ET}}(j-1)}=0\right)$ then the relative ramp-rate is computed in terms of $p_{\min }^{i}$. If generator $i$ is off at $\mathrm{PTU} \kappa_{\mathrm{ET}}(j)$ there is no need to impose ramp-rate constraints. Finally, (6f)-(6h) are the energy balances inside each PTU of the prediction horizon. Notice that special care needs to be taken for the last PTU, $\bar{n}(k)$, specifically when the end of PTU $\bar{n}(k)$ does not coincide with the end of the prediction horizon (see $(6 a))$. In that case, we scale the required $e^{\text {final }}(\bar{n}(k))$ proportionally to the length of the PTU that is inside the prediction horizon. This percentage is given by $s_{k}^{N_{\mathrm{E}}} \triangleq \bmod \left(\frac{\left(k+N_{\mathrm{E}}\right) T_{\mathrm{E}}}{T_{\mathrm{PTU}}}\right) / T_{\mathrm{PTU}}$. Notice that the upperlevel MPC problem (6) is a convex quadratic program for which efficient, off-the-shelf software exist for its solution. 


$$
\begin{aligned}
& \min \sum_{j=k}^{k+N_{\mathrm{E}}-1} \ell_{\mathrm{p}}(\bar{p}(j))+\sum_{n=\underline{n}(k)}^{\bar{n}(k)-1} \ell_{\mathrm{IM}}\left(\bar{e}^{\mathrm{P}}(n)+\bar{e}^{\mathrm{rd}}(n)-\bar{e}^{\mathrm{final}}(n)\right)+\ell_{\mathrm{IM}}\left(s_{k}^{N_{\mathrm{E}}}\left(\bar{e}^{\mathrm{P}}(\bar{n}(k))+\bar{e}^{\mathrm{rd}}(\bar{n}(k))\right)-\bar{e}^{\mathrm{final}}(\bar{n}(k))\right) \\
& \text { s.t. } \bar{p}(k-1)=p^{\mathrm{av}}(k-1), \bar{e}^{\mathrm{p}}(\underline{n}(k))=e^{\mathrm{p}}(\underline{n}(k) ; k), \bar{e}^{\mathrm{rd}}(\underline{n}(k))=e^{\mathrm{rd}}(\underline{n}(k) ; k), \bar{e}^{\mathrm{final}}(\underline{n}(k))=e^{\mathrm{final}}(\underline{n}(k)) \text {, } \\
& \mathbf{I}_{i, \kappa_{\mathrm{ET}}(j)} \bar{p}_{\min }^{i} \leq \bar{p}^{i}(j) \leq \mathbf{I}_{i, \kappa_{\mathrm{ET}}(j)} \bar{p}_{\max }^{i}, \\
& i \in \mathbb{N}_{\left[1, n_{\mathrm{p}}\right]}, j \in \mathbb{N}_{\left[k, k+N_{\mathrm{E}}-1\right]}, \\
& \frac{T_{\mathrm{E}}}{60} \Delta p_{\min }^{i} \leq \frac{\bar{p}^{i}(j)-\bar{p}^{i}(j-1)}{\bar{p}^{i}(j-1)} \leq \frac{T_{\mathrm{E}}}{60} \Delta p_{\max }^{i}, \\
& \frac{T_{\mathrm{E}}}{60} \Delta p_{\min }^{i} \leq \frac{\bar{p}^{i}(j)-p_{\min }^{i}}{p_{\min }^{i}} \leq \frac{T_{\mathrm{E}}}{60} \Delta p_{\max }^{i}, \\
& \bar{e}^{\mathrm{p}}(\underline{n}(k))=\bar{e}^{\mathrm{P}}(\underline{n}(k))+\frac{T_{\mathrm{E}}}{3600} \sum_{j=k}^{k_{\max }} \sum_{i=1}^{(k)} \sum_{\overline{\mathrm{p}}}^{n_{\mathrm{p}}}(j), \bar{e}^{\mathrm{rd}}(\underline{n}(k))=\bar{e}^{\mathrm{rd}}(\underline{n}(k))+\frac{T_{\mathrm{E}}}{3600} \sum_{j=k}^{k_{\max }(\underline{n}(k))}\left(\sum_{i=1}^{n_{\mathrm{r}}} \bar{r}^{i}(j)-\bar{d}(j)\right), \\
& \bar{e}^{\mathrm{P}}(n)=\frac{T_{\mathrm{E}}}{3600} \sum_{j=k_{\min }(n)}^{k_{\max }(n)} \sum_{i=1}^{n_{\mathrm{p}}} \bar{p}^{i}(j), \bar{e}^{\mathrm{rd}}(n)=\frac{T_{\mathrm{E}}}{3600} \sum_{j=k_{\min }(n)}^{k_{\max }(n)}\left(\sum_{i=1}^{n_{\mathrm{r}}} \bar{r}^{i}(j)-\bar{d}(j)\right), \quad n \in \mathbb{N}_{[\underline{n}(k)+1, \bar{n}(k)-1]}, \\
& \bar{e}^{\mathrm{p}}(\bar{n}(k))=\frac{T_{\mathrm{E}}}{3600} \sum_{j=k_{\min }(\bar{n}(k))}^{k+N_{\mathrm{E}}-1} \sum_{i=1}^{n_{\mathrm{p}}} \bar{p}^{i}(j), \quad \bar{e}^{\mathrm{rd}}(\bar{n}(k))=\frac{T_{\mathrm{E}}}{3600} \sum_{j=k_{\min }(\bar{n}(k))}^{k+N_{\mathrm{E}}-1}\left(\sum_{i=1}^{n_{\mathrm{r}}} \bar{r}^{i}(j)-\bar{d}(j)\right), \\
& \bar{e}^{\mathrm{final}}(\underline{n}(k))=\bar{e}^{\mathrm{final}}(\underline{n}(k))+\sum_{j=k}^{k_{\max }(\underline{n}(k))} \bar{e}^{\mathrm{sc}}(j), \\
& \bar{e}^{\mathrm{final}}(n)=\bar{e}^{\operatorname{prog}}(n)+\sum_{j=k_{\min }(n)}^{k_{\max }(n)} \bar{e}^{\mathrm{sc}}(j), \\
& \bar{e}^{\mathrm{final}}(\bar{n}(k))=\bar{e}^{\mathrm{prog}}(\bar{n}(k))+\sum_{j=k_{\min }(\bar{n}(k))}^{k+N_{\mathrm{E}}-1} \bar{e}^{\mathrm{sc}}(j)
\end{aligned}
$$

\section{POWER TIME SCALE}

\subsection{Dynamics of Controllable Generators}

In this paper, the model developed in [Roffel and de Boer, 2003 ] is used to describe the dynamics of the generators. The model consists of two parts, a fast model for primary reserve action and a slow model part for the secondary reserve activation. The model representing the relatively fast power changes consists of a low and a high pass filter connected in series:

$$
p_{\text {fast }}^{i}(s)=\frac{\tau_{\mathrm{H}}^{i} s}{\tau_{\mathrm{H}}^{i} s+1} \frac{K^{i}}{\tau_{\mathrm{L}}^{i} s+1} p_{\text {prim }}^{i}(s),
$$

where $p_{\text {prim }}^{i}$ is the power for primary control. The slow model is given by

$$
p_{\text {slow }}^{i}(s)=\frac{e^{-T_{\text {delay }}^{i} s}}{\tau^{i} s+1}\left(u^{i}(s)+p_{\text {prim }}^{i}(s)\right),
$$

where $u^{i}$ is the power setpoint of the generator (manipulated variable). Primary control is described by

$$
p_{\text {prim }}^{i}(s)=-\frac{100 p_{\max }^{i}}{f_{\text {nom }} \operatorname{droop}^{i}} \delta f(s),
$$

where $\delta f$ is the change in frequency $[\mathrm{Hz}]$ (measured disturbance), $f_{\text {nom }}$ is the nominal frequency $(50 \mathrm{~Hz})$, droop $^{i}$ is the droop (or statism) of the generator in \%. Finally, the power output of generator $i$ is given by

$$
p^{i}(s)=p_{\text {fast }}^{i}(s)+p_{\text {slow }}^{i}(s) \text {. }
$$

Using a first-order Padé approximation for the time-delay and a $\mathrm{ZOH}$ discretization with sampling time equal to $T_{\mathrm{P}}$ for the continuous-time system, a minimal state-space realization of (7)-(9) is given by

$$
\begin{aligned}
x^{i}(t+1) & =A^{i} x^{i}(t)+B^{i} u^{i}(t)+E^{i} \delta f(t), \\
p^{i}(t) & =C^{i} x^{i}(t),
\end{aligned}
$$

where $y^{i}(t)=\left[p_{\text {slow }}^{i}(t) p^{i}(t)\right]^{\prime}, x^{i} \in \mathbb{R}^{4}$ are the $i$-th generator's output and state vector. The aggregated dynamics of the $n_{\mathrm{p}}$ generators of the BRP can be written in the compact form

$$
\begin{aligned}
x(t+1) & =A x(t)+B u(t)+E \delta f(t), \\
p(t) & =C x(t) .
\end{aligned}
$$

\subsection{Lower-level MPC}

The lower-level MPC acts on the power time--scale, its goal being to track the reference power and energy signals of the energy time-scale obtained by the upper-level MPC scheme. Let $N_{\mathrm{P}}$ denote the prediction horizon of the MPC controller operating on the energy time-scale. Then, the energy time intervals that are active during the prediction horizon, starting from the power time interval $t$, are $k \in$ $\mathbb{N}_{[\underline{k}(t), \bar{k}(t)]}$, where $\underline{k}(t) \triangleq \kappa_{\mathrm{PE}}(t)$, and $\bar{k}(t) \triangleq \kappa_{\mathrm{PE}}\left(t+N_{\mathrm{P}}\right)$. At every $t \in \mathbb{Z}_{+}$on the power time scale, the lower level $\mathrm{MPC}$ tries to track the most recently obtained reference signals by the upper level MPC.

Specifically, the lower level MPC scheme uses as setpoints the power and energy sequences obtained by the most recently solved upper level MPC. We use the notation $\bar{e}_{k}^{\mathrm{p}}$, $p_{j}^{i}, x_{j}, \delta f_{j}$ to denote predictions for $e_{k}^{\mathrm{p}}, p^{i}(j), x(j), \delta f(j)$. Hence, the lower level MPC solves the optimal control problem (13)-(27) at every time $t \in \mathbb{Z}_{+}$on the power time-scale. Since a full state measurement is not available, we use an estimation $\hat{x}(t)$ of the true state vector of the generators at time $t, x(t)$, obtained through an observer (e.g., a Kalman filter).

Equation (14) provides the initial conditions (current power injections and estimated states of the generators) while (15) describes the dynamics of the controllable generators. Here, $\delta f_{j}$ can be considered constant along the prediction horizon $\left(\delta f_{j}=\delta f(t)\right)$, or a point forecast can be used instead. Equations (16), (17), (18) are bound and ramp-rate constraints on the inputs (power setpoints), respectively. For the power output of the generators we impose bound and ramp-rate constraints on $p_{\text {slow }}$ but not 


$$
\begin{aligned}
& \min \sum_{j=t}^{t+N_{\mathrm{P}}}\left\|p_{j}-\bar{p}\left(\kappa_{\mathrm{PE}}(j)\right)\right\|_{Q}^{2}+\sum_{k=\underline{k}(t)}^{\bar{k}(t)} q_{k}\left(\Delta e_{k}\right)^{2}+\sum_{j=t+1}^{t+N_{\mathrm{P}}}\left(\underline{r}_{j}\left\|\underline{\epsilon}_{j}\right\|^{2}+\bar{r}_{j}\left\|\bar{\epsilon}_{j}\right\|^{2}+\underline{\delta r_{j}}\left\|\underline{\delta \epsilon_{j}}\right\|^{2}+\overline{\delta r}_{j}\left\|\overline{\delta \epsilon_{j}}\right\|^{2}\right) \\
& \text { s.t. } p_{t}=p(t), \delta f_{t}=\delta f(t), x_{t}=\hat{x}(t) \text {, } \\
& x_{j+1}=A x_{j}+B u_{j}+E \delta f_{j}, p_{j+1}=C_{1} x_{j+1}, p_{\text {slow }, j+1}=C_{2} x_{j+1}, \\
& \mathbf{I}_{i, \kappa_{\mathrm{PT}}(j)} p_{\min }^{i} \leq u_{j}^{i} \leq \mathbf{I}_{i, \kappa_{\mathrm{PT}}(j)} p_{\max }^{i}, \\
& j \in \mathbb{N}_{\left[t, t+N_{\mathrm{P}}-1\right]}, \\
& \frac{T_{\mathrm{P}}}{60} \Delta p_{\text {min }}^{i} \leq \frac{u_{j}^{i}-u_{j-1}^{i}}{u_{j-1}^{i}} \leq \frac{T_{\mathrm{P}}}{60} \Delta p_{\text {max }}^{i}, \\
& \mathbf{I}_{i, j-1}=1, \mathbf{I}_{i, j}=1, i \in \mathbb{N}_{\left[1, n_{\mathrm{p}}\right]}, j \in \mathbb{N}_{\left[t, t+N_{\mathrm{P}}-1\right]} \text {, } \\
& \frac{T_{\mathrm{P}}}{60} \Delta p_{\min }^{i} \leq \frac{u_{j}^{i}-p_{\min }^{i}}{p_{\min }^{i}} \leq \frac{T_{\mathrm{P}}}{60} \Delta p_{\max }^{i}, \\
& \mathbf{I}_{i, \kappa_{\mathrm{PT}}(j)}\left(p_{\min }^{i}-\underline{\epsilon}_{j}^{i}\right) \leq p_{\text {slow }, j}^{i} \leq \mathbf{I}_{i, \kappa_{\mathrm{PT}}(j)}\left(p_{\max }^{i}+\bar{\epsilon}_{j}^{i}\right), \\
& \mathbf{I}_{i, j-1}=0, \mathbf{I}_{i, j}=1, i \in \mathbb{N}_{\left[1, n_{\mathrm{p}}\right]}, j \in \mathbb{N}_{\left[t, t+N_{\mathrm{P}}-1\right]}, \\
& i \in \mathbb{N}_{\left[1, n_{\mathrm{p}}\right]}, j \in \mathbb{N}_{\left[t+1, t+N_{\mathrm{P}}\right]},
\end{aligned}
$$

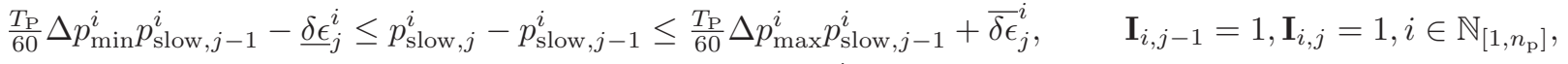

$$
\begin{aligned}
& \frac{T_{\mathrm{P}}}{60} \Delta p_{\min }^{i} p_{\min }^{i}-\underline{\delta \epsilon_{j}^{i}} \leq p_{\mathrm{slow}, j}^{i}-p_{\min }^{i} \leq \frac{T_{\mathrm{P}}}{60} \Delta p_{\max }^{i} p_{\min }^{i}+\overline{\delta \epsilon}_{j}^{i},
\end{aligned}
$$

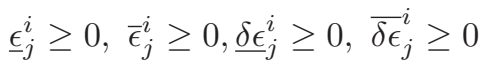

$$
\begin{aligned}
& \bar{e}_{\underline{k}(t)}^{\mathrm{p}}=e_{\underline{k}(t)}^{\mathrm{p}}(t)+\frac{T_{\mathrm{P}}}{3600} \sum_{j=t}^{t_{\max }(\underline{k}(t))} \sum_{i=1}^{n_{\mathrm{p}}} p_{j}^{i}, \\
& \bar{e}_{k}^{p}=\frac{T_{\mathrm{P}}}{3600} \sum_{j=t_{\min }(k)}^{t_{\max }(k)} \sum_{i=1}^{n_{\mathrm{p}}} p_{j}^{i}, \\
& \bar{e}_{\bar{k}(t)}^{p}=\frac{T_{\mathrm{P}}}{3600} \sum_{j=t_{\min }(\bar{k}(t))}^{t+N_{\mathrm{P}}-1} \sum_{i=1}^{n_{\mathrm{p}}} p_{j}^{i}, \\
& \Delta e_{k}=\bar{e}_{k}^{\mathrm{p}}-\bar{e}^{\mathrm{p}}\left(\kappa_{\mathrm{ET}}(k) ; k+1\right), \\
& \Delta e_{\bar{k}(t)}=\bar{e}_{\bar{k}(t)}^{\mathrm{p}}-s_{t}^{N_{\mathrm{P}}} \bar{e}^{\mathrm{P}}\left(\kappa_{\mathrm{ET}}(\bar{k}(t)) ; \bar{k}(t)+1\right) .
\end{aligned}
$$

on $p_{\text {fast }}$ since no limits are imposed on fast response of the generators. These limitations on $p_{\text {slow }}$ are expressed as soft constraints to prevent infeasibility of the optimization problem in real-time. This is done by adding (subtracting) slack variables $\bar{\epsilon}_{j}^{i}$ and $\overline{\delta \epsilon}_{j}^{i}\left(\underline{\epsilon}_{j}^{i}\right.$ and $\left.\underline{\delta \epsilon}_{j}^{i}\right)$ on the right hand side (left hand side) of (19)-(21). Equations (23)-(25) describe the energy balances in the power time scale. In $(23), e_{\underline{k}(t)}^{\mathrm{p}}(t)$ is already known and can be calculated using the power injections of the BRP on the power time-scale so far.

The cost function in eq. (13) penalizes deviations from the power and energy setpoints. Here $Q$ is a positive definite matrix, while $q_{k}, k \in \mathbb{N}_{[\underline{k}(t), \bar{k}(t)]}$ are positive scalars. These can be decreasing with $k$ since the goal is to put more emphasis on energy tracking for the current PTU and take advantage of good short term predictions. However one also would like to penalize energy tracking error for future PTUs since penalization of the energy setpoints provides a form of integral action for the lower level MPC. This is because trying to track the power setpoints only may not be sufficient for E-Program fulfillment, since the power injections on the power time scale need some time to reach the setpoints, due to the ramp-rate constraints. Again, the last term of the energy setpoint is scaled appropriately multiplying by $s_{t}^{N_{\mathrm{P}}} \triangleq \bmod \left(\frac{\left(t+N_{\mathrm{P}}+1\right) T_{\mathrm{P}}}{T_{\mathrm{E}}}\right) / T_{\mathrm{E}}$. Finally, the weighted sum of the norms of output constraint violations is penalized in the cost function. The power set-points that are applied to generators of the BRP at every time $t \in \mathbb{Z}_{+}$ are $u^{i}(t)=u_{t}^{i}, i \in \mathbb{N}_{\left[1, n_{\mathrm{p}}\right]}$.

\section{CASE STUDY}

The proposed MPC scheme is tested on a BRP consisting of 10 controllable generators, most of which are Combined
Table 1. Comparison of HMPC and SPT

\begin{tabular}{lrrr}
\hline Controller & Generation & Imbalance & Total cost \\
\hline \hline HMPC & $578,674 €$ & $-49,057 €$ & $529,617 €$ \\
SPT & $549,257 €$ & $-3,285 €$ & $545,973 €$ \\
\hline
\end{tabular}

Cycle Gas Turbines, and one uncontrollable generator (wind farm). In the simulations undertaken, realistic data for wind generation, wind forecast, E-Program, imbalance prices, frequency deviation, volume of AS bids activated in real-time are used to test and evaluate the performance of the MPC algorithm. The data were obtained from KEMA and the website of TenneT. The simulation was carried out for 16 PTUs $\left(T_{\mathrm{PTU}}=15 \mathrm{~min}\right)$. The energy sampling time is $T_{\mathrm{E}}=60 \mathrm{~s}$, while the power sampling time is $T_{\mathrm{P}}=4 \mathrm{~s}$. The prediction horizon for the upperlevel MPC is $N_{\mathrm{E}}=16$, while for the lower-level MPC is $N_{\mathrm{P}}=10$. BRP data are omitted due to space limitations. Perturbed versions of the realizations for the uncertain parameters $d(t), r(t), e^{\mathrm{sc}}(t), \delta f(t), \lambda_{\mathrm{IM}}^{ \pm}(n)$ are used as predictions in the MPC scheme, to test the tolerance of the MPC scheme to prediction errors. The proposed HSMC scheme was compared against an algorithm which reflects current practice to some extend, that is, power setpoints for the generators for each PTU are computed by the Unit Commitment module in an open-loop fashion, on the dayahead, while in real-time the controller tries to reach the setpoints along the current PTU in a static fashion. We call this algorithm Static set-Point Tracking (SPT).

In Table 1 the two schemes are compared with respect to total simulation cost along the 16 PTUs. Clearly, HMPC exhibits superior performance to SPT mainly due to its predictive ability regarding uncontrollable generation and imbalance prices. 


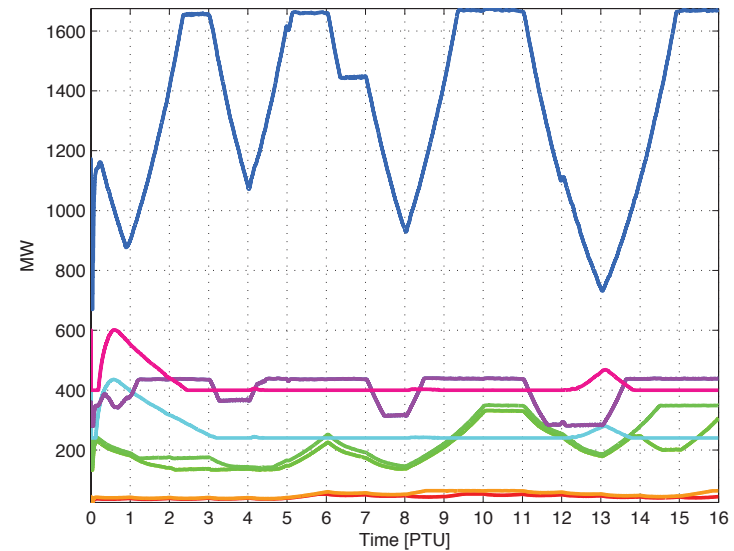

Fig. 1. Power profile of BRP plants for HMPC.

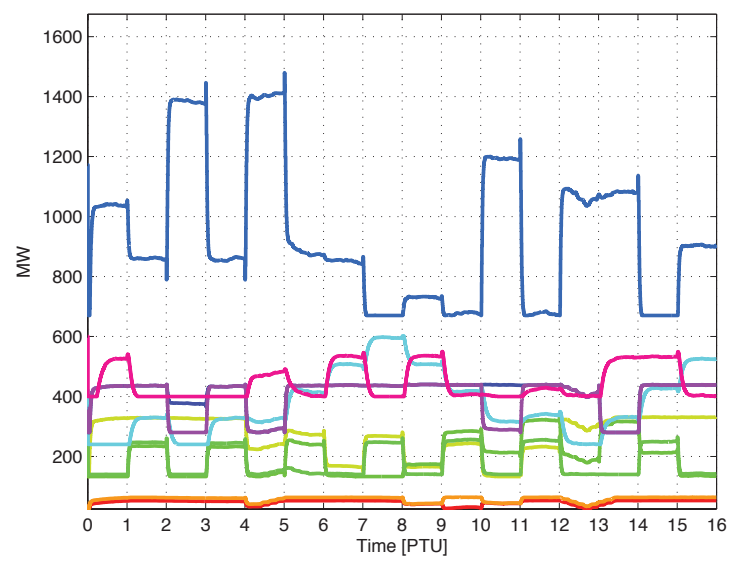

Fig. 2. Power profile of BRP plants for SPT.

Figures 1 and 2 depict the power profiles of the generators for HMPC and SPT, respectively. The abrupt changes on the power profiles for SPT (fig. 2) clearly shows its inability to handle ramp-rate constraints and allow smooth transitions between PTUs. On the other hand, due to explicit ramp-rate constraint handling and energy integral action, HMPC exhibits smooth transitions between PTUs, as shown in fig. 1 .

\section{CONCLUSIONS AND FUTURE WORK}

The "Smart Grid" concept mainly aims at introducing renewable sources in the power grid, diversifying electricity generators and reducing environmental pollution by considering a new kind of player within energy markets, the so called Balance Responsible Parties. Starting from this point, this work has provided an innovative control scheme for real-time operation of BRPs, based on hierarchical model predictive control. The approach provides a reliable and valid solution for integration of renewable energy sources, solving their most crucial problems related to intermittence and forecast errors, by computing in realtime economically optimal set-points based on accurate predictions. An important aspect of the algorithm is that it improves the quality in electricity distribution services, since it takes into account requests provided by TSO, assisting it in secondary control, and consequently helping TSO to manage the security of the electrical network. Further research plans include extending the algorithm into a stochastic framework using results of [Patrinos et al., 2011], so as to further improve performance and robustness properties of the controller, as well as incorporating priceelastic prosumers into the BRP model.

\section{ACKNOWLEDGEMENTS}

The authors would like to thank Gerben Dekker (KEMA) for his invaluable comments.

\section{REFERENCES}

A. Jokic. Price-based Optimal Control of Electrical Power Systems. PhD thesis, Technische Universiteit Eindhoven, 2007.

P. Patrinos, S. Trimboli, and A. Bemporad. Stochastic MPC for real-time market-based optimal power dispatch. In 50th IEEE Conf. on Decision and Control and European Control Conf., Orlando, FL, pages 71117116, 2011.

B. Roffel and W.W. de Boer. Analysis of power and frequency control requirements in view of increased decentralized production and market liberalization. Control engineering practice, 11(4):367-375, 2003.

M. Sewalt and C. de Jong. Negative prices in electricity markets. Commodities Now-Online journal: www. commodities-now. com, 2003.

M. Shahidehpour, H. Yamin, and Z. Li. Market operations in electric power systems. Wiley, 2002.

B.C. Ummels, M. Gibescu, E. Pelgrum, W.L. Kling, and A.J. Brand. Impacts of wind power on thermal generation unit commitment and dispatch. IEEE Transactions on Energy Conversion, 22(1):44-51, 2007. 